\title{
Contextual influences on the interaction between conflicting memories in the rat
}

\author{
NORMAN E. SPEAR, GREGORY J. SMITH, RICHARD G. BRYAN, \\ and WILLIAM C. GORDON \\ State University of New York, Binghamton, New York 13901
}

and

ROBIN TIMMONS and DAVID A. CHISZAR

Rutgers University, New Brunswick, New Jersey 08903

\begin{abstract}
Contextual events redundant to the learning, in sequence, of passive and active avoidance were of one type for the former task and of another for the latter. During later testing, these contextual events were found to determine which of these acquired, conflicting dispositions would be manifested in behavior. Mutual interference in retention otherwise seen under similar circumstances seemed subordinate to the influence of the three types of contextual stimuli tested-drug (pentobarbital), compound (the experimental room in which conditioning took place), and a relatively unitary stimulus (a constantly sounding buzzer). The discussion considered the defining characteristics of a "contextual stimulus" and the mechanisms through which contextual events control the manifestation of learned behaviors and alleviation of interference in retention.
\end{abstract}

The role of contextual cues in basic conditioning and learning has interested researchers for the past 50 years (Asratian, 1965; Konorski, 1967; Pavlov, 1927). It has been recognized that, generally speaking, the conditioning procedure probably leads not only to the formation of associations between the CS and the UCS, but also to associations between the experimental situation and the CS/UCS, and that the excitability of the CS units may be enhanced in the context in which conditioning had taken place (e.g., Konorski, 1967, chap. 6). Recent experiments have provided important new leads about the influence of contextual stimuli on the conditioned performances of human and animals (e.g., Kamin, 1969; Kimmel \& Ray, 1978; Pearce \& Hall, 1979; Tomie, 1980), and conditioning theory has now implicated contextual influences as central in elementary associative learning (e.g., Rescorla \& Wagner, 1972; Wagner, 1976).

"Contexts" of either a linguistic or nonlinguistic variety have also become an increasingly frequent consideration of theorists concerned with human or animal memory (Estes, 1976; Smith, Glenberg, \&

Preparation of this article was supported by grants from the National Science Foundation (BNS 7424194 and BNS 78 02360) to the first author. We would especially like to thank Norman Richter for technical advice and assistance and Teri Tanenhaus for preparation of the manuscript. Requests for reprints should be sent to Dr. Norman E. Spear, Department of Psychology, State University of New York at Binghamton, Binghamton, New York 13901. R. G. Bryan is now at Keuka College; W. C. Gordon is now at the University of New Mexico; and D. A. Chiszar is now at the University of Colorado.
Bjork, 1978; Spear, 1978). For example, retention of prior learning is aided when certain features of the learning context are present, even though these same features were redundant to original learning. In our view, this effect of contextual cues may be accomplished in either of two ways: (1) by facilitating the discrimination between equally accessible dispositions or memories of prior experiences; or (2) by promoting retrieval of an otherwise inaccessible memory (Spear, 1976). The present paper initiates the experimental analysis of these two alternatives in an avoidance learning paradigm with rats.

There is an established interaction between sequentially learned passive and active avoidance responses. Rats that learn to run from place A to place B in oneway active avoidance training perform more poorly when retested on this task later if they had previously learned to stay in place A during passive avoidance training (e.g., Gordon, Frankl, \& Hamberg, 1979; Spear, 1971; Spear, Gordon, \& Chiszar, 1972). The present experiments examined the ability of a variety of contextual stimuli to reduce the interaction between these learning experiences. We investigated internal drug-induced stimuli (Experiment 1), nonspecific exteroceptive stimuli (Experiment 2), and a relatively specific exteroceptive stimulus (Experiment 3) as contextual cues in the sequential avoidance-training task.

In Experiments 2 and 3, we also attempted to distinguish between the "discriminative" and "retrieval" functions of contextual stimuli by testing the relative effects of these stimuli after different lengths of 
time following training. We reasoned that if contextual stimuli enhance the discrimination between two equally accessible memories, the contextual cue should be most effective shortly after both memories have been acquired (when either memory can be accessed readily) and progressively less effective as accessibility declines after longer intervals. Alternatively, if the contextual cue serves a retrieval function, its effect might be greater after longer intervals. The rationale for this alternative can be stated briefly. Soon after learning, the animal has available an abundance of cues similar to those present during learning. These may include, for example, the sensory aftereffects, sequential events, and hormonal and neurochemical states that are maintained through original learning and persist into a retention test given soon afterward. These temporarily dominant cues may continue to arouse corresponding attributes of the most recently acquired memory and thus cause, shortly after learning, a momentarily inflated probability of retrieving the target attribute of that memory (i.e., manifestation of the index of learning). However, these transient "short-term-memory" cues are likely replaced or modified after longer intervals, when more remote contextual cues may increase in importance relative to other potential retrieval cues. This principle has been applied as one possible explanation for the increasing interaction between go and no-go memories that accompanies longer retention intervals (Spear, 1971, 1978; Spear et al., 1972).

\section{EXPERIMENT 1}

In this experiment, we assessed whether the interaction between two conflicting behaviors could be controlled by correlated differences in internal context induced by injection of pentobarbital. Two groups of rats were given passive-shock-avoidance training to avoid the black compartment of an avoidance apparatus by remaining in the alternative, white compartment. One group was trained following injection of pentobarbital, and the other, following injection of saline. Twenty-four hours after acquiring this passive avoidance, these same rats were trained on the contrary behavior-to approach rapidly the black compartment (active shock avoidance). During active avoidance, the drug states were reversed from those of the previous day's training; rats given passive avoidance training under saline were given active avoidance training under pentobarbital, and vice versa. Finally, $24 \mathrm{~h}$ after acquiring active avoidance, half the rats were injected with pentobarbital and half with saline before being given five nonshock test trials. The test trials were conducted exactly as were passive and active avoidance training, but with neither response-shock contingency. If the rat could discriminate between these two conflicting behaviors on the basis of the internal contextual states present during the training phase, then the competition between these two behaviors should be minimal. Otherwise, under the present conditions of a relatively long intertask interval, the more recently acquired response (active avoidance) should dominate performance 24 h later (Gordon \& Spear, 1973; Spear, 1971).

\section{Method}

Subjects. Forty-eight experimentally naive, male albino SpragueDawley rats born and raised in our colony were used in this experiment. These rats ranged in age from 60 to 80 days and were housed in steel-mesh laboratory cages in a climate-controlled vivarium with ad-lib food and water and constant illumination.

Apparatus. The apparatus was a two-chamber black-and-white avoidance apparatus housed in a dimiy lighted room. Each chamber $(26.5 \mathrm{~cm}$ long, $14.5 \mathrm{~cm}$ high, and $12.5 \mathrm{~cm}$ wide, one black, one white) rested on a grid floor that could be electrified by a GrasonStadler shock generator and scrambler (Model E1064 GS). Separating each compartment was an opaque door which, when lowered, formed a $2.5-\mathrm{cm}$ hurdle and initiated a $2 \cdot \mathrm{Hz}$ flashing light and a latency timer. Upon jumping the hurdle, the rat broke a photocell beam in the black compartment that terminated the flashing light and timer.

Injection procedure. Twenty minutes prior to training and testing, the rats were injected intraperitoneally with pentobarbital (Nembutal, $15 \mathrm{mg} / \mathrm{kg} / \mathrm{ml}$ ) or an equal volume of $.9 \%$ saline. To accustom the rats to the injection procedure, all rats were weighed and injected once daily over a 4-day period prior to training, twice with saline and twice with pentobarbital, in a counterbalanced fashion.

Training procedure. In Phase I, half the rats received passive avoidance training, while the other half received an equivalent amount of handling. Within each treatment, half the rats were injected with pentobarbital and the other half with saline. For passive avoidance training, the rat was first placed in the white compartment of the apparatus facing away from the door. Approximately $3 \mathrm{sec}$ later, the door was lowered and the rat was allowed access to the black compartment. Upon crossing over into the black compartment, the rat broke a photocell beam which initiated a 3-sec, 1.6-mA inescapable footshock through the grid floor. The rat was then removed from the black compartment and placed in a holding cage for $30 \mathrm{sec}$ to await the next training trial. If the rat failed to cross over into the black compartment within $60 \mathrm{sec}$, an avoidance was counted and the rat was returned to the holding cage. Passive avoidance training continued until the rat withheld crossing over into the black compartment on three consecutive trials plus five additional avoidances. Rats in the handled control condition were carried to the experimental room and placed in the holding cage, from which they were picked up and returned approximately five times.

Twenty-four hours after the completion of Phase I, all rats were given active avoidance training (Phase II) except that rats given saline during Phase I were now injected with pentobarbital and rats originaliy jnjected with pentobarbital were given saline. Active avoidance was identical to that for passive avoidance except, of course, for the response-footshock contingencies. The rat was first placed in the white compartment of the apparatus facing away from the door. When the apparatus door was lowered, the rat avoided footshock if it stepped across into the black compartment within $5 \mathrm{sec}$. A failure to step across into the black compartment within $5 \mathrm{sec}$ initiated a 1.6-mA footshock delivered through the grid floor in the white compartment. The shock remained on until the rat escaped to the black compartment. The rat was immediately taken out of the black compartment and placed into a holding cage for $30 \mathrm{sec}$ to await the next trial. Active avoidance training continued until the rat made three consecutive avoidances plus five additional avoidances. 
Testing procedure. All testing occurred 23-24 h after completion of Phase II. During testing, each rat was given five nonshock trials. Half the rats were tested in the same drug state as that of Phase II, while the other half were tested in the drug state present during Phase I.

Design. This experiment employed a 2 by 2 by 2 factorial design2 Prior Treatments (passive avoidance training or handling) by Drug State During Active Avoidance Training by Drug State During Testing - with six rats assigned randomly to each of eight orthogonal treatment groups. These data were analyzed with analysis of variance tests, with $p<.05$ as the criterion for rejection of the null hypothesis.

\section{Results and Discussion}

Acquisition of passive avoidance (PA). The mean numbers of trials to complete the PA learning criterion for rats trained following injection of pentobarbital $(\bar{X}=10.17)$ or of saline $(\bar{X}=9.67)$ were not reliably different.

Acquisition of active avoidance (AA). Rats injected with pentobarbital during this phase required 20.1 trials to complete the criterion as opposed to 15.6 trials for rats injected with saline. This main effect of drug during AA was reliable $[F(1,40)=9.55]$; no other sources of variance in trials to criterion achieved statistical significance in this phase. While this common effect of pentobarbital on rate of acquiring the AA may conceivably imply a difference in terminal levels of learning that could contaminate conclusions about the absolute effect of AA drug on retention, our present questions concern conclusions to be derived from interactions, not this particular main effect.

Finally, analysis of the first-trial crossover latencies during AA indicated that rats previously given PA training exhibited longer crossover latencies ( $\overline{\mathrm{X}}=$ $8.56 \mathrm{sec})$ than those previously only handled $[\bar{X}=$ $5.73 \mathrm{sec}, F(1,40)=14.09]$. This effect and the $a b$ sence of its interaction with drug state suggest that the drug-induced dissociation was incomplete, but this was, nevertheless, a transient effect not observed on subsequent AA trials.

Retention test. When the drug present at testing matched that present during AA learning, response latencies tended to be short; but when the drug at testing matched that present during PA learning, response latencies tended to be long. In other words, retention test performance was in accord with the requirements of the task previously associated with the testing drug. This general pattern of results occurred in terms of responding both on the first test trial and throughout all test trials combined.

Among rats that had learned both active and passive avoidance, those tested in the drug state that had existed during passive avoidance learning exhibited longer mean crossover latencies throughout testing $(\bar{X}=47.16 \mathrm{sec})$ than rats tested in the drug state corresponding to active avoidance learning $(\bar{X}=10.59 \mathrm{sec}$; see Figure 1, lower panel). Analogous differences are
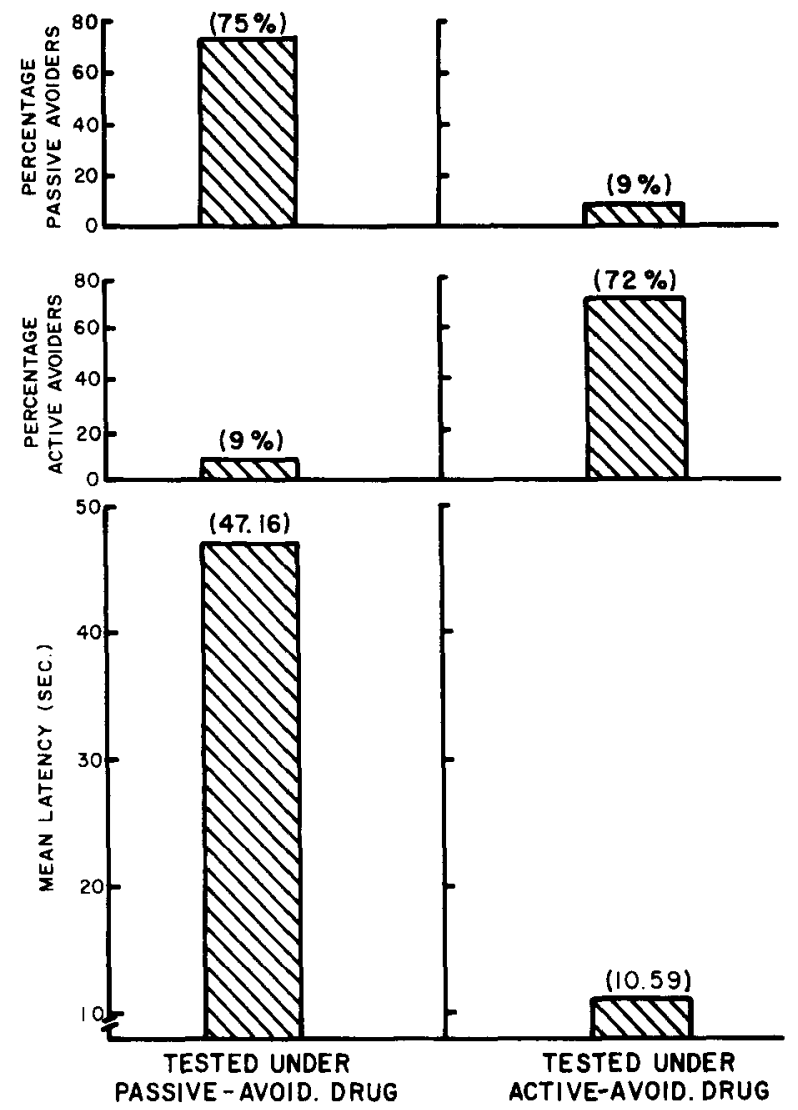

Figure 1. The results of the retention test in Experiment 1 are shown separately for animals tested under the drug state associated with passive avoidance learning (saline or pentobarbital) and those tested under that present during active avoidance learning. The bottom panel shows mean latency over all five test trials. The top and middle panels indicate the percentage of animals in each condition that behaved in accord with the criterion for passive avoidance and active avoidance, respectively, on more than half of the test trials.

reflected in the top two panels of Figure 1. Here it can be seen that $75 \%$ of all rats tested in the same drug state that existed during PA learning exhibited PA behavior (failure to cross over into the black compartment within $60 \mathrm{sec}$ on at least three of the five test trials) as opposed to only $9 \%$ making AA responses (latencies less than $5 \mathrm{sec}$ on at least three of the five test trials). A similar finding was observed for rats tested in the same drug state that existed during AA learning. Here, $72 \%$ of these rats showed a marked tendency for AA behavior while only $9 \%$ showed PA behavior.

Statistical tests confirmed these obvious differences. Rats tested in the same drug state that existed during PA learning exhibited reliably slower crossover latencies both on Trial 1 and Trials 1-5 (combined) than animals tested in the drug state corresponding to that of AA learning $[F s(1,40)=8.46$ and 13.66, respectively]. This effect tended to occur for both those animals actually trained on PA and those 
given only equivalent handling, although of course with larger magnitude among the former animals. This is reflected in the marginally significant interaction between type of experience prior to AA learning (PA or handling) and similarity between the drug states of training and testing [for Test Trial 1 and Trials $1-5$, respectively, $F s(1,40)=3.18$ and 3.89 , $\mathrm{p}<.10]$. Apparently, the tendency for slightly longer latencies among animals given pentobarbital, the imposition of a 60 -sec ceiling on the response measure, and an influence of simple state-dependent learning for animals trained only on active avoidance acted in opposition to more statistically robust confirmation of this interaction. Finally, while on the first test trial there was a significant main effect of drug during test due to the general "slowing" effect of pentobarbital $[F(1,40)=5.57]$, this difference was not reliable for the remaining test trials.

This experiment establishes the feasibility of the present PA-AA transfer paradigm for assessing the expression of conflicting dispositions for behaviors acquired in different contexts. These results suggest that the reinstatement of the internal physiological drug state (e.g., pentobarbital vs. saline) during testing may aid the discrimination between the alternative behaviors. This is not the only interpretation suggested by these data. It is conceivable, for instance, that during training under the drug state the animal may come to rely on stimuli different from those that control behavior in the nondrug state and subsequently influence test performance independent of context.

Was the acquired disposition (or "'memory") for passive avoidance behavior disintegrated or altered in the direction of a weakened tendency as a consequence of learning active avoidance, or vice versa? It would appear that, under the present circumstances, the answer would be no. The integrity of the disposition for each of these conflicting behaviors seemed to be rather sharply maintained, in that its behavioral manifestation in the same context under which it was acquired reflects little interaction with the alternative (cf. Spear et al., 1972). The next step was to assess the generality of this effect in terms of a different class of contextual stimuli, one that would permit variation in temporal parameters of theoretical interest.

\section{EXPERIMENT 2}

Having demonstrated, in Experiment 1, the feasibility of the PA-AA transfer paradigm in assessing the effects of training context on test performance, Experiment 2 sought to demonstrate similar effects by manipulating external features in the environment. The external environmental features selected for manipulation were the particular room and apparatus used for training or testing. We expected this external contextual stimulus to have a strong effect on the animal's behavior when changed between training and testing but, unlike pentobarbital, to have minimal direct performance effects that might contaminate the index of learning. Furthermore, the external contextual cue could be terminated abruptly, unlike the drug-induced internal context of Experiment 1 . This permitted orthogonal variation in length of retention interval in the present experiment as well as a within-subject test of the influence of contextual stimuli on test performance, and so provided a preliminary test of whether context acts primarily to "facilitate discrimination" or to "promote retrieval" (Spear, 1976).

\section{Method}

Generally, the apparatus, learning tasks, parameters of training, learning criteria, and sequencing of learning tasks were identical to those of Experiment 1.

Subjects. The subjects were 64 experimentally naive, female albino Sprague-Dawley rats, between 75 and 90 days of age, from the same source and housed and maintained as in Experiment 1. Four rats were assigned randomly to each of the 16 groups in this experiment.

Training procedure. The training procedures and specific parameters for AA and PA were identical to those used in Experiment 1 , although no drug injections were administered. Instead of the drug state cue, this experiment manipulated the room in which training occurred. These were two rooms (room $X$ and room Y) located adjacent to one another in the same building and approximately equal in size. They were equipped with identical avoidance apparatuses (see Experiment 1 for a description of the apparatus). No attempt was made to equate the rooms in terms of furniture arrangements, lighting, odors, etc. ${ }^{\text {' }}$

In Phase I, half the rats received PA training or equivalent handling in room $X$ and the other half received identical treatment in room $Y$. Three minutes after completion of Phase I experience, all rats were given AA training (Phase II).

In Phase II, those rats given Phase $I$ in room $X$ had AA train ing in room $Y$ and those given Phase $I$ in room $Y$ had $A A$ training in room $X$.

Testing procedure. The retention test occurred either 3 min or $24 \mathrm{~h}$ after the completion of Phase II. All rats were given five nonshock test trials in one of two rooms (X or $Y$ ), followed $3 \mathrm{~min}$ later by five additional nonshock test trials in the alternative ( $Y$ or $\mathrm{X}$ ). The order in which rooms were chosen and assigned was random and counterbalanced within all groups.

Design. A 2 by 2 by 2 by 2 factorial experimental design was employed. The variable of major interest was the contextual cue, which, in this experiment, was simply one of the two possible experimental rooms. The four variables were: the specific experimental room used for active avoidance training (room $\mathrm{X}$ or room $\mathrm{Y}$ ); the room used for the five trials of initial testing ( $\mathrm{X}$ or $\mathrm{Y}$, followed by five test trials in the alternative room); Phase I experience (PA training or only equivalent handling); and retention interval following attainment of the criterion of AA learning ( 3 min or $24 \mathrm{~h}$ ).

\section{Results}

Acquisition. Among the 16 groups in this experiment, no differences occurred in the number of trials required to attain the criterion for either $\mathrm{PA}$ or $\mathrm{AA}$ learning (all $F s<1$ ). The mean number of trials to complete the PA learning criterion was 9.5 , while the 


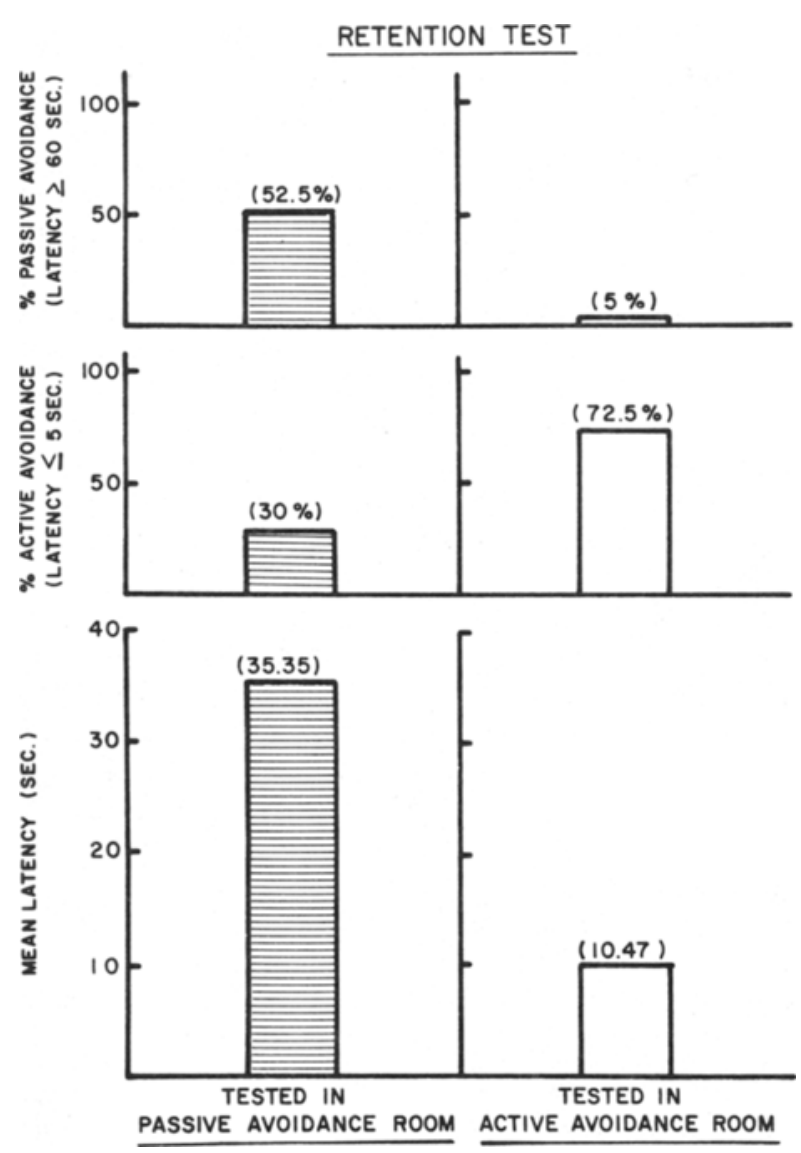

Figure 2. The results of the retention test in Experiment 2 are shown separately for animals tested in the room used for passive avoidance learning and those tested in the room used for active avoidance learning. The bottom panel shows mean latency over all five test trials. The top and middle panels indicate the percentage of animals in each condition that behaved in accord with the criterion for passive avoidance and active avoidance, respectively, on more than half of the test trials.

AA learning criterion was completed in a mean of 15.2 trials. In addition, the particular room in which rats were trained had no absolute effects on performance. All subjects may therefore be considered equal in terms of their degree of learning prior to the retention test. As in Experiment 1, crossover latencies on the first active avoidance training trial were significantly slower for those animals receiving previous passive avoidance training than for those only handled $[\bar{X}=7.59$ and $5.13 \mathrm{sec}$, respectively; $F(1,56)$ =35.6]. Again, as in Experiment 1, this was a transient difference not observed on any subsequent active avoidance training trials or accompanied by differences in rate of learning.

Retention test. The results of both retention tests are shown in Figure 2. As depicted in this figure, the left panel in each graph represents test performance in the PA (passive avoidance) training room and the right panels represent test performance in the AA (active avoidance) training room.
The four-way analysis of variance performed on mean crossover latency during testing indicated the predicted three-way interaction between room context, prior (Phase I) treatment, and testing blocks $[\mathrm{F}(1,112)=11.35]$, which will be discussed shortly. Another general result of this analysis that should be mentioned at this point is the absence of any statistically reliable main effects or interactions involving the retention interval factor. This is important, because a decision between the theoretical alternatives emphasizing discrimination or retrieval depends on the influence of the retention interval, to at least some extent, and so it will require further consideration. For now, however, all figures presented in this section represent data pooled across retention intervals, and we will not discuss this factor further in this section.

As seen in Figure 2, the general effect in this experiment was in complete accord with that of Experiment 1: considering all 10 retention test trials, rats tested in the room where they had learned PA exhibited significantly longer crossover latencies than the rats tested in the room where they had acquired AA $[\mathrm{F}(1,56)=6.03]$.

In Figure 3, the test results are broken down according to first retention test (Trials $1-5$ ) and second retention test (Trials 6-10). Note that half the rats were tested first in the PA room and tested second in the AA room, and the others were tested in the opposite order. The results of these tests will be considered separately.

In terms of their performance on the first test (Tri-

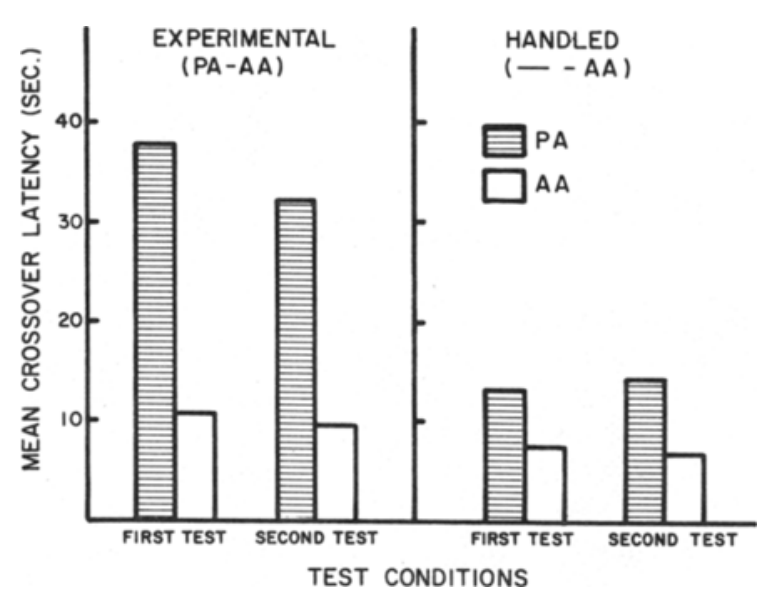

Figure 3. Mean crossover latencies during the retention test in Experiment 2 are shown separately for animals given passive avoidance training prior to learning active avoidance and for those given only equivalent handling prior to learning active avoidance. Subjects were given their first tests either in the room where they had received their prior treatments (PA room) or where they had received their active avoidance training ( $A A$ room); the second test for each subject was given in the alternative room. Thus, in each of the two panels in this figure, animals represented by $P A$ in the first test are represented by $A A$ in the second test, and vice versa. 
als 1-5), the rats tested in the PA (or handling) room showed significantly longer latencies than those tested in the AA room $[F(1,56)=9.32]$. This difference was significantly more pronounced for rats actually given PA than for those merely handled in the PA room, as confirmed by the interaction between testing room and treatment in the PA room prior to $\mathrm{AA}[\mathrm{F}(1,56)=$ 4.14]. This suggests that the contextual cues controlled manifestation of each of the PA and AA memories, or perhaps permitted their discrimination, rather than affecting only that for AA.

As seen in Figure 3, the differential control exerted by the particular testing room was maintained when rats were tested in the room opposite that of their first retention test. With reversal of the room of testing, there was a corresponding reversal in test behavior. Rats given a second test in the room previously used for PA training or handling exhibited longer crossover latencies throughout this test than did rats tested in the room where they had previously been given AA training $[F(1,56)=5.75]$; although not shown in Figure 3, a subsequent control experiment confirmed our expectation that animals given the training sequence used in Experiment 2 but all 10 test trials in one room do not show a comparable shift in performance between Test Trials 1-5 and 6-10. The effect of context tended again to be greater for rats given prior PA training than for those merely handled in the PA room, although statistical significance for this interaction was only marginal across the last 5 test trials $[F(1,56)=3.56, p<.10]$.

To summarize, the behavior of rats in the experimental groups tested in one room during Trials 6-10 was essentially opposite that exhibited by these same rats just 3 min earlier in the alternative room (Trials 1-5). Animals that previously had exhibited slow crossover latencies when the room context was similar to prior PA training exhibited fast latencies when the room context corresponded to that of AA training. The opposite effect occurred among those subjects tested initially in the AA context and later in the PA context. Each of these effects was greater among subjects given actual PA training than it was among those given only equivalent handling prior to $\mathrm{AA}$ training, as confirmed by the significant triple interaction between similarity of training- and testingroom context, prior treatment, and testing block [Trials $1-5$ vs. $6-10 ; \mathrm{F}(1,112)=11.35$ ]. Furthermore, the order in which these rooms were used during testing and the specific rooms themselves did not modulate behavior independent of conditioning.

\section{Discussion}

In this experiment, the general context of the training room was sufficient for eliciting the specific behaviors acquired in those rooms. Rats trained to avoid the black compartment in room $\mathrm{X}$ (passive avoidance, PA) and to avoid the white compartment in room Y (active avoidance, AA) selectively performed those behaviors upon returning to the individual rooms, regardless of whether tested first in room X or room Y. Experiment 2 obtained no evidence that this effect of context depends on length of the retention interval, however.

Several experiments with procedures like those of Experiment 2 have shown clearly that the interaction between the memories representing the conflicting PA and AA episodes is relatively slight immediately after their acquisition (the most recently acquired memory dominates behavior), although it is substantial later in time (e.g., Gordon, Frankl, \& Hamberg, 1979; Spear et al., 1972). One way to view such a time-dependent interference effect is in terms of a "protection interval," which, for a short period following new learning, has the property of effectively blocking the influence of stimuli that might otherwise promote retrieval of previously acquired, conflicting memories (Spear, 1971, 1978). It was therefore expected that, for Experiment 2, we would find that imposition of the context associated with prior PA learning would have relatively little effect on test performance shortly after AA learning, in comparison with the control that contextual cue would exert on test performance $24 \mathrm{~h}$ later. The results of Experiment 2 did not concur with this prediction.

\section{EXPERIMENT 3}

In Experiment 3, the exteroceptive contextual stimulus was intended to be relatively unitary and perhaps less pervasive than that of Experiment 2, and some procedural changes were introduced to provide a more sensitive test of the effect of retention interval. Rats were given either PA training or only equivalent handling in either the presence or the $a b$ sence of a constantly sounding buzzer (the contextual stimulus). Because we hoped to decrease the interaction between prior and most-recent learning on later retention when the contextual stimulus was absent, and thus permit more sensitive measurement of the greater interaction expected when the contextual stimulus had been present during prior learning, lesser PA training was given than in Experiments 1 and 2. One minute after attaining the PA learning criterion, each rat was given AA training. After reaching the AA learning criterion, the rats were given five nonshock test trials $3 \mathrm{~min}, 15 \mathrm{~min}, 30 \mathrm{~min}$, $60 \mathrm{~min}, 4 \mathrm{~h}, 24 \mathrm{~h}$, or 21 days later, always in the presence of the sounding buzzer. If the effectiveness of the contextual stimulus is not affected by length of the retention interval, the disruption in retention of AA caused by prior PA learning should be enhanced to an equal degree among animals given PA in the context of the sounding buzzer, irrespective of time since AA. 


\section{Method}

Subjects and apparatus. For this experiment we used 140 rats, 80-100 days of age. Males and females were apportioned about equally among the 28 experimental conditions. These rats were from the same source and were housed and maintained as in Experiments 1 and 2. The apparatus was identical to that of Experiments 1 and 2 .

Training procedure. The training procedure was identical to that used in Experiments 1 and 2. Thus, on each trial of PA training, AA training, or testing, the rat was placed into the white compartment of the apparatus and, approximately $3 \mathrm{sec}$ later, the apparatus door was lowered and a $2-\mathrm{Hz}$ flashing light illuminated the white compartment.

In Phase I, as in Experiments 1 and 2, half the rats received passive-shock-avoidance (PA) training while the other half were given an equivalent amount of handling. Unlike Experiments 1 and 2 , half the rats in each of these treatments were exposed to a constantly sounding $\mathrm{BD}-1$ Line dc buzzer from the moment the rat entered the experimental room until $30 \mathrm{sec}$ after completion of PA or handling. PA training continued until the rat failed to step across into the black compartment within $60 \mathrm{sec}$ on two consecutive training trials.

In Phase II, 1 min after completion of Phase I, each rat was given AA training until it achieved the criterion of three consecutive avoidances (crossover latencies less than $5 \mathrm{sec}$ ) in the same experimental room used for $\mathbf{P h a s e} \mathrm{I}$.

Testing procedure. Retention tests, conducted in the same apparatus used for training, were given $3 \mathrm{~min}, 15 \mathrm{~min}, 30 \mathrm{~min}$, $60 \mathrm{~min}, 4 \mathrm{~h}, 24 \mathrm{~h}$, or 21 days after completion of Phase II. Each rat was returned to its home cage in the vivarium between AA and testing. Each rat was given a total of five nonshock test trials like those in the previous experiments but in the presence of a constantly sounding buzzer.

\section{Results and Discussion}

Acquisition. In Phase I (PA), analysis of variance performed on the first-trial crossover latency indicated no differences in latencies for rats exposed to the buzzer and those not exposed $[F(1,56)=1.26]$. Most rats $(86 \%)$ attained the PA criterion in one trial while the remainder required two or three trials. Analysis of variance performed on trials to attain criterion indicated no differences among the 14 groups given PA training.

Acquisition. In Phase II (AA), the transient influence of PA training on AA learning again was noticeable only on the first trial. On this trial, rats given prior PA training took longer to escape the white (shocked) compartment than did handled controls $[\mathrm{F}(1,112)=34.2]$. On subsequent trials, as in the previous experiments, no reliable differences in crossover latencies were observed $(F<1)$. Rats with prior PA training required 11.18 trials and rats previously given only handling required 11.04 trials prior to criterion; neither this difference nor any other differences with this measure approached statistical significance among the experimental conditions. This suggests that all rats in this experiment had learned active avoidance equally well prior to the retention interval.

Retention test. Generally, the tendency to perform the more recently acquired behavior (AA) decreased at a faster rate over time for animals that had had prior PA training than for those without it. This agrees with previous results (Gordon et al., 1979; Spear, 1971; Spear et al., 1972). Furthermore, the former effect was magnified for rats given both prior PA training and the retention test within the context of the buzzer sound.

Crossover latencies on the first trial of the retention test are shown in Figure 4. Among rats for whom the buzzer had been part of the context of Phase I treatment (upper panel, Figure 4), greater AA deficits were observed for those animals given prior passive avoidance learning than for those only handled $[F(1,56)=36.66]$ and for those animals given the longer retention intervals $[F(6,56)=6.55]$. The main effects of these variables must be qualified, however, due to the significant interaction between them $[F(6,56)=5.48]$. This interaction confirms that

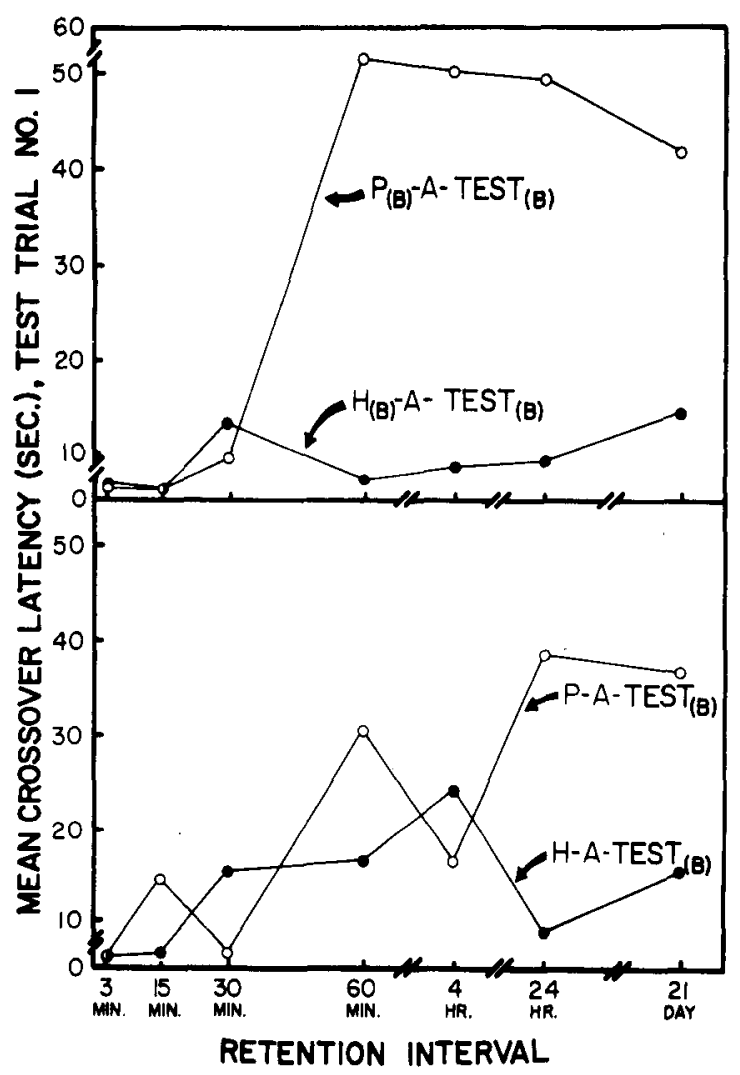

Figure 4. Mean latency to cross over from the white to the black compartment is shown for separate groups of animals tested after one of seven intervals following the learning of active avoidance (A). Prior to learning the active avoidance, animals either learned passive avoidance $(P)$ or received an equivalent amount of handling $(H)$. The presence of the buzzer as a contextual cue is indicated by $\mathrm{B}$; all subjects received their tests in the presence of the buzzer, while those represented in the upper panel also received their passive avoidance or equivalent handling in the presence of the buzzer. For example, the open circles in the upper panel represent the scores for subjects that first learned passive avoidance in the presence of the buzzer, then learned active avoidance in the absence of the buzzer, then were tested in the presence of the buzzer. 
the contribution of prior PA learning to forgetting AA was greater after longer intervals. For those animals not exposed to the apparatus-buzzer combination prior to the retention test (lower panel, Figure 4), these effects of prior learning and retention interval did not attain statistical significance on the first trial, perhaps due to the relatively low degree of passive avoidance learning in this experiment (cf. Gordon et al., 1979; Spear et al., 1972) or to disruptive consequences of the animal's first exposure to the buzzer in the apparatus context.

Supplementary retention measures generally agree with response latency on the first test trial in showing that, for all animals, forgetting of active avoidance was increased by prior passive avoidance learning and longer retention intervals. Analysis of the combined data from all five retention test trials, in terms of either mean crossover latency or mean number of trials on which a "passive avoidance" occurred (i.e., on which the rat did not cross over within the 60-sec time limit), indicated a significant interaction between Phase I training (PA vs. only handling) and retention interval-greater effect of prior training the longer the retention interval-for only those animals exposed to the buzzer during Phase I as well as the retention test $[F(6,56)=6.75$ for mean crossover latency; and $F=7.97$ for number of passive avoidances]. Those animals not exposed to the buzzer during their Phase I experience showed no such significant interaction ( $\mathrm{F}<1$ for mean crossover latency; and $\mathrm{F}=1.58$ for number of passive avoidances).

The general conclusion that emerges from these results is that prior PA learning in the context of the buzzer had a marked decremental effect on AA test performance. Furthermore, this decrement in test performance was enhanced the longer the time since training. The conservative test of these assertions, however, would require a triple interaction between presence of buzzer during prior experience (Phase I), type of Phase I experience, and length of the retention interval. Such an interaction, while apparent numerically, did not achieve statistical significance for any of the measures employed as indexes of retention. Thus, it cannot be firmly concluded at this time that the effects of contextual cues (in selectively determining which of two conflicting behaviors is to be manifested) depend upon length of the retention interval following latest learning.

\section{GENERAL DISCUSSION}

Experiment 1 demonstrated that an otherwise redundant internal drug state (e.g., pentobarbital vs. saline) paired on a single occasion with the training of one of two competing avoidance behaviors would subsequently elicit the specific response ac- quired in the context of that drug state. Experiment 2 replicated this basic finding using different, external environmental stimuli (e.g., experimental conditioning room) as the cues associated with conflicting behaviors, and found similar effects after either short or long retention intervals. Experiment 3 extended the findings of the previous experiments in examining the control of contextual stimuli on test performance at various intervals after training. The results of Experiment 3 indicated that there might be a period of time shortly after training when access to the memory of the most recently acquired behavior is impervious to disruption.

These experiments indicate the considerable control of alternative learned behaviors that may be exerted by contextual stimuli, even when these stimuli apparently were redundant to the requirements of original learning. The presence of these contextual events during testing can result in manifestation of a corresponding memory that is surprisingly intact, in the sense that the behavior observed reflects quite faithfully the effects of the particular training episode that had appeared in that context. This may be contrasted with the alternative possibility-behavior which, due to the animal's exposure to a directly conflicting episode, is inappropriate in spite of the testing context. The present study therefore emphasizes that the integrity of learned behavior, and perhaps also the entire memory of a learning episode, may be maintained in spite of the acquisition of a severely conflicting memory.

Yet, by way of a caveat, it seems clear that with the present procedures the estimate of "maintained integrity" of a memory is quite rough. It is important to derive more precise comparisons of the structure of a memory when a conflicting one is, and is not, acquired. The present procedures represent one general technique for activation of one or the other of two conflicting memories that may now be exploited in the investigation of retroactive and proactive interference in animals. A variety of similar techniques that also test the characteristics of contextual stimuli have been employed by Thomas, Welker, Tomie, and their colleagues to good advantage with the pigeon (e.g., Tomie, 1976, 1980; Welker, Tomie, Davitt, \& Thomas, 1974) and by Smith, Glenberg, and Bjork (1978) and others with humans.

We have not yet undertaken the thoroughly systematic investigation needed to study the properties of contextual cues as a function of retention interval. It is notable, in this respect, that we have conducted in our laboratory a substantial number of (unpublished) experiments toward developing a suitable experimental paradigm to permit an appropriately sophisticated experimental analysis of the effect. To date, we have been unsuccessful in obtaining a sufficiently robust "protection interval" phenomenon 
with the present go, no-go procedures to permit this analysis.

Does a contextual stimulus act by facilitating discrimination among equally accessible memories or by directly promoting retrieval of the memory with which it is associated? The discrimination view would be supported if the influence of the contextual cue were strongest relatively recently after memory acquisition, when both memories are most likely accessible, and then waned with decreasing accessibility. We found no evidence for this relationship. Although retention after the short interval was influenced by the contextual stimulus in Experiment 2, there were indications in Experiment 3 that the contextual stimulus was rather ineffective shortly after acquisition of the conflicting memory and more effective later on. Support for the alternative view-direct promotion of retrieval by the contextual cue-was also equivocal in the present study. In this case, however, the direction of the result and the less conservative statistical tests (Experiment 3) were supportive. Also, it is possible that statistical support in terms of the more conservative test was precluded by the ceiling placed on measurement in Experiment 3 or that this effect is seen only with retention intervals shorter than $24 \mathrm{~h}$. It would appear premature to discard either of the alternative theories.

\section{REFERENCES}

Asratian, E. A. Compensatory adaptations, reflex activity in the brain. Oxford: Pergamon Press, 1965.

Estes, W. K. (ED.), Handbook of learning and cognitive processes (Vol. 4) Attention and memory. Hillsdale, N.J: Erlbaum, 1976.

Gordon, W. C., \& Spear, N. E. The effects of strychnine on a recently acquired and reactivated passive avoidance memory. Physiology \& Behavior, 1973, 10, 1071-1075.

Gordon, W. C., Frankl, S. E., \& Hamberg, J. M. Reactivationinduced proactive interference in rats. American Journal of Psychology, 1979, 92, 693-702.

Kam IN, L. J. Predictability, surprise, attention, and conditioning. In B. A. Campbell \& R. M. Church (Eds.), Punishment and aversive behavior. New York: Appleton-Century-Crofts, 1969.

Kimmel, H. D., \& RAY, R. L. Transswitching: Conditioning with tonic and phasic stimuli. Journal of Experimental Psychology: General, 1978, 107, 187-205.

KonorSKI, J. Integrative activity of the brain-An interdisciplinary approach. Chicago: University of Chicago Press, 1967.

Pavlov, I. P. Conditioned reflexes (T. V. Anrep, trans.). London: Oxford University Press, 1927.
Pearce, J. M., \& Hall, G. The influence of context-reintorcer associations on instrumental performance. Animal Learning \& Behavior, 1979, 7, 504-508.

Rescorla, R. A., \& Wagner, A. R. A theory of Pavlovian conditioning: Variations in the effectiveness of reinforcement and nonreinforcement. In A. H. Black \& W. F. Prokasy (Eds.), Classical conditioning 1I: Current research and theory. New York: Appleton-Century-Crofts, 1972.

Smith, S. M., Glenberg, A., \& Bjork, R. A. Environmental context in human memory. Memory \& Cognition, 1978, 6, $342-353$.

Spear, N. E. Forgetting as retrieval failure. In W. K. Honig \& P. H. R. James (Eds.), Animal memory. New York: Academic Press, 1971.

Spear, N. E. Retrieval of memories: A psychobiological approach In W. K. Estes (Ed.), Handbook of learning and cognitive processes (Vol. 4) Memory processes. Hillsdale, N.J: Erlbaum, 1976.

Spear, N. E. The processing of memories: Forgetting and retention. Hillsdale, N. J: Erlbaum, 1978.

Spear, N. E., Gordon, W. C., \& Chiszar, D. A. Interaction between memories in the rat: Effect of degree of prior conflicting learning on forgetting after short intervals. Journal of Comparative and Physiological Psychology, 1972, 78, 471-477.

TOMIE, A. Interference with autoshaping by prior context conditioning. Journal of Experimental Psychology: Animal Behavior Processes, 1976, 2, 323-334.

TOMIE, A. Effects of unpredictable food upon the subsequent acquisition of autoshaping: Analysis of the context-blocking hypothesis. In C. M. Locurto, H. S. Terrace, \& J. Gibbon (Eds.), Autoshaping and conditioning theory. New York: Academic Press, 1980.

Wagner, A. R. Priming and STM: An information processing mechanism for self-generated or retrieval-generated depression in performance. In T. J. Tighe \& R. N. Leaton (Eds.), Habituation: Perspectives from child development, animal behavior, and neurophysiology. Hillsdale, N.J: Erlbaum, 1976.

Welker, R. L., Tomif, A., Davitt, G. A., \& Thomas, D. R. Contextual stimulus control over operant responding in pigeons. Journal of Comparative and Physiological Psychology, 1974, 86, 549-562.

\section{NOTE}

1. The same experimenter conducted training and testing in each of the two rooms. Among the notable differences in objects in the two rooms, a straight-alley runway was present in one and a group of four operant chambers in the other. None of these auxiliary apparatuses were in operation at the time of training or testing in the present experiments. Although the two rooms had no distinctive differences in odor that could be detected by humans, and although dim lighting was maintained at about the same levels in both rooms, it seems plausible that differences in either of these conditions could have been detected by the rats.

(Received for publication December 22, 1978; revision accepted October $22,1979$. 